\title{
Possible Measurable Effects of Dark Energy in Rotating Superconductors
}

\author{
Clovis Jacinto de Matos ${ }^{1}$ and Christian Beck ${ }^{2}$ \\ ${ }^{1}$ ESA-HQ, European Space Agency, 8-10 rue Mario Nikis, 75015 Paris, France \\ ${ }^{2}$ School of Mathematical Sciences, Queen Mary, University of London, Mile End Road, London E1 4NS, UK
}

Correspondence should be addressed to Christian Beck, c.beck@qmul.ac.uk

Received 16 March 2009; Accepted 26 June 2009

Recommended by Zdzislaw E. Musielak

We discuss recent laboratory experiments with rotating superconductors and show that three so far unexplained experimentally observed effects (anomalous acceleration signals, anomalous gyroscope signals, Cooper pair mass excess) can be physically explained in terms of a possible interaction of dark energy with Cooper pairs. Our approach is based on a Ginzburg-Landaulike model of electromagnetic dark energy, where gravitationally active photons obtain mass in the superconductor. We show that this model can account simultaneously for the anomalous acceleration and anomalous gravitomagnetic fields around rotating superconductors measured by Tajmar et al. and for the anomalous Cooper pair mass in superconductive Niobium, measured by Cabrera and Tate. It is argued that these three different physical effects are ultimately different experimental manifestations of the simultaneous spontaneous breaking of gauge invariance and of the principle of general covariance in superconductive materials.

Copyright ( $\odot 2009$ C. J. de Matos and C. Beck. This is an open access article distributed under the Creative Commons Attribution License, which permits unrestricted use, distribution, and reproduction in any medium, provided the original work is properly cited.

\section{Introduction}

The existence of dark energy in the universe, as indicated by numerous astrophysical observations, represents one of the most challenging problems in theoretical physics at present [1-4]. A great variety of different models exist for dark energy but none of these models can be regarded as being entirely convincing so far. The cosmological constant problem (i.e., the smallness of the cosmologically observed vacuum energy density) remains an unsolved problem. It is likely that the solution of this problem requires new, so far unknown, physics.

While it is clear that dark energy has measurable effects on cosmological scales (such as the accelerated expansion of the universe as seen from supernovae observations), it is much less clear what the effects of dark energy could be on smaller scales. These effects, if any, depend very much on the model considered. For example, if dark energy is due to the existence of compactified dimensions with a diameter of the order of the micron scale, then this would lead to modifications of the gravitational interaction potential on these scales. This can be tested in laboratory precision experiments. Tests by Kapner et al. [5] proved negative so far down to a scale of about 50 microns.

Other more recent models of dark energy, such as the electromagnetic dark energy model of Beck and Mackey [6], also produce potentially measurable effects at laboratory scales, which are, however, restricted to the interior of superconductors. In [6] a Ginzburg-Landau theory is constructed that generates a cutoff for the gravitational activity of vacuum fluctuations. Generally it is assumed in this model that vacuum fluctuations of any particle can exist in two different phases: a gravitational active one (contributing to the cosmological constant $\Lambda$ ) and a gravitationally inactive one (not contributing to $\Lambda$ ). The model exhibits a phase transition at a critical frequency which makes the dark energy density in the universe small and finite. The above approach has many analogies with the physics of superconductors, and in particular it allows for a possible interaction between dark energy and Cooper pairs. A suppression of the cosmological constant due to the formation of quantum condensates has also been discussed in [7].

In models of dark energy like the above one it is the new physics underlying the cutoff that can potentially lead 
to measurable effects in the laboratory (see also [8] for related work). In Beck and Mackey's model [6] dark energy couples to superconducting matter only (and not to matter in the normal state). This is theoretically consistent. If we assume that dark energy can interact with superconducting matter only, we do not get any contradiction from cosmological observations, since almost all of the matter in the universe is not in a superconducting state. Given the above assumption of a possible interaction between dark energy and superconducting matter one can then constrain the interaction strength by making precision measurements with superconducting devices.

In this paper we look at recent experiments that were performed with rotating superconductors. There are three observed anomalies that cannot be explained with conventional theories. One dates back already 20 years. Tate et al. [9] made precision measurements of the London moment in rotating superconductors. The London moment is a magnetic field generated inside a superconductor once it is set into rotation. They measured a London moment slighty too large as compared to the theoretical expectations. This anomalous London moment, well established within the experimental precision, has remained unexplained for the past 20 years. More recently, Tajmar et al. [10] investigated rotating superconductors using isolated laser gyroscopes positioned outside the superconductor. The gyroscopes yield small signals proportional to the rotation frequency that again cannot be explained by conventional theories. These signals may, however, be interpreted in terms of a gravitomagnetic field whose strength is much larger than theoretically expected from ordinary gravity. Finally, Tajmar et al. [11] also measured anomalous induced acceleration signals in isolated accelerometers close to a rotating superconductor, which occur if the rotation frequency of the superconductor is rapidly changed. Again this induced acceleration signal is much stronger than theoretically expected from normal gravity. All three effects are specific to superconducting matter, they vanish as soon as the temperature exceeds a critical temperature. Presently it seems that none of the above measured effects can be understood in terms of conventional superconductor physics.

We will show that all three effects can be quantitatively understood by assuming a possible interaction between Cooper pairs and dark energy as described in the model of Beck and Mackey [6]. The key for a quantitative understanding of the experimental observation is the fact that photons formally obtain mass in a superconductor, due to a finite London penetration depth. In particular, gravitationally active photons whose vacuum fluctuations underly dark energy in the model [6] obtain mass as well and lead effectively to a strong enhancement of gravitoelectromagnetic effects, which can be experimentally measured. Ultimately, our theoretical interpretation is that not only gauge invariance but also general covariance is spontaneously broken in the superconducting material, the latter one being related to an interaction of dark energy with Cooper pairs.

This paper is organized as follows. In Sections 2 and 3 we briefly summarize the experimentally observed effects in rotating superconductors that could possibly be linked to dark energy. In Sections 4-6 we develop the tools for our theoretical approach, based on the Einstein-MaxwellProca equations describing gravitomagnetic fields and the electromagnetic dark energy model of Beck and Mackey. Finally, in Sections 7-9 we show that our theory can explain the experimentally observed effects in a quantitatively correct way.

\section{Tajmar's Experiments}

Tajmar et al. have established a research programme at Austrian Research Centers GmbH-ARC with the objective of peering into new possible gravitational properties of superconductive materials. Two different types of experiments have been carried out.

(1) The first category provides measurements of azimuthal accelerations with accelerometers located inside the equatorial plane of the central hole of different types of superconductive rings, which are angularly accelerated. The experimental arrangement was designed to ensure a minimal thermal and mechanical coupling between the accelerometers and the ring's motion. The accelerometers were isolated as much as possible from the whole experimental setup inside a sealed vacuum chamber $[10,11]$.

(2) In the second category, angular velocities were measured with laser gyroscopes located above different types of uniformly rotating superconductive rings (with respective equatorial planes parallel to each other). Again the overall experimental setup and the measurement methods ensured that the laser gyros were maximally decoupled from undesired mechanical torques in general, and from the ring's motion in particular $[10,12]$.

Based on the known laws of physics, and taking into account all known physical effects in the experimental setup described above in (1) and (2), neither the accelerometers nor the laser gyros should indicate any significant signal above the noise level. This is not what Tajmar's experiments demonstrated. Rather, an azimuthal acceleration signal, which could be associated with an anomalous gravitational field, directly proportional to the superconductive ring angular acceleration, and an angular velocity signal orthogonal to the ring's equatorial plane, which could be associated with an anomalous gravitomagnetic field, have been measured in type- 1 and type- 2 experiments, respectively $[10,11]$.

\subsection{Acceleration Fields Around Angularly Accelerated Rotating} Superconductors. In Tajmar's experiment, the superconducting ring has a radius of $R=0.07 \mathrm{~m}$ and the accelerometers are positioned inside the central hole of the ring at distance $r=0.036 \mathrm{~m}$. In the case of Niobium rings, the measured coupling between the applied angular acceleration $\dot{\omega}$ to the superconducting ring (measured in $\mathrm{rad} / \mathrm{s}^{2}$ ) and the measured induced azimuthal acceleration $g$ inside the central hole of the ring has the following value and associated error (see [11, Table 2]):

$$
\frac{g}{\dot{\omega}}=-(9.46 \pm 0.28) \times 10^{-7} \mathrm{~m} \cdot \operatorname{Rad}^{-1}
$$


If the angular frequency is measured in units of $\left[s^{-1}\right]$, this result is equivalent to

$$
\frac{g}{\dot{\omega}}=-(1.51 \pm 0.04) \times 10^{-7} \mathrm{~m} .
$$

Like the gravitational field produced by the mass of a physical body, the measured acceleration, $g$, does not depend on the accelerometer's mass or chemical composition detecting it. The effect is only seen if the ring is in the superconductive state, that is, below its respective critical temperature $T_{c}$, and if the ring is accelerated. The coupling as given by (2) depends on the ring's material type, and disappears, within the instrumentation resolution capability, for High- $T_{c}$ superconductors.

The above value of the coupling is based on single-sensor measurements and the evaluation of maximum acceleration peaks [11]. If multiple sensors at different positions are used (in a so-called curl configuration) and if an average signal analysis is performed, then smaller coupling constants are obtained [10]. However, for the physical interpretation we are going to provide in Section 7 (in terms of a particle emission event) the maximum peak analysis with a single sensor is the most appropriate one.

Acceleration peaks were also observed when the superconductor passed through its critical temperature while it was rotating at constant angular velocity. These signals had opposite signs for the transition from the normal to the superconductive state and vice versa.

What could be the origin of the measured anomalous acceleration inside the central region of an angularly accelerated Niobium superconductive ring? What could account for the measured coupling (2)? Before we answer these questions let us first investigate the anomalous gravitomagnetic properties of uniformly rotating superconductors.

\subsection{Gravitomagnetic Fields Around Uniformly Rotating Super-} conductors. In the limit of small field strengths and for nonrelativistic movements, the Einstein equations yield a set of Maxwell-like equations which describe so-called gravitomagnetic fields [13]. For normal matter these gravitational fields are much weaker than electromagnetic fields. However, in coherent quantum systems the experiments seem to provide evidence for much stronger than expected gravitomagnetic fields [14].

The most recent experiments of Tajmar et al. use laser gyroscopes to detect gravitomagnetic fields. A laser gyroscope is an interferometer, measuring the phase difference between two beams of coherent electromagnetic waves with equal frequency, $\nu_{0}$, propagating in opposite directions along a closed optical fiber. When the fiber rotates with angular velocity $\Omega$, a phase difference, $\delta \phi$, is measured. This is the so-called Sagnac effect

$$
\delta \phi=4 \nu_{0} \frac{S \Omega}{c^{2}} .
$$

Here $S$ is the component of the optical fiber cross-section parallel to the rotation plane.

The same effect, a phase difference, can be caused by a gravitomagnetic flux crossing a laser gyroscope at rest, since a gravitomagnetic field $\vec{B}_{g}$ originates from a vector potential $\vec{A}_{g}$ :

$$
\vec{B}_{g}=\nabla \times \vec{A}_{g}
$$

In that case the phase difference is directly proportional to the intensity of the gravitomagnetic field:

$$
\delta \phi=4 v_{0} \frac{S B_{g}}{c^{2}} .
$$

Here $S$ is the component of the optical fiber cross-section orthogonal to the gravitomagnetic field. Such a phase difference is measured by Tajmar et al. $[10,12]$ when a Niobium superconductive ring is rotating with constant angular velocity $\omega$, with an isolated laser-gyroscope positioned nearby. The measured coupling $\chi^{\prime}$ between the gravitomagnetic field and the angular velocity of the superconductive Niobium ring is of the order

$$
\chi^{\prime}=\frac{B_{g}}{\omega} \sim 10^{-8}
$$

\section{Cabrera and Tate's Measurements}

Superconductors at rest expell any magnetic field. But when they exhibit rotational motion, a magnetic field $\vec{B}$ is generated within the superconductor, the so-called London moment

$$
\vec{B}=-2 \frac{m}{e} \vec{\omega} .
$$

Here $m$ and $e$ are the mass and charge of the Cooper pair. This effect is not accounted for by classical electrodynamics, it can only be properly explained in the framework of quantum field theory [15]. It consists in the spontaneous generation of a magnetic field by setting a superconductor into rotation in an environment initially (before the rotation starts) entirely free from any electromagnetic fields.

By measuring the $\vec{B}$ field very precisely, one can conclude on the Cooper pair mass $m$ using (7). The experimental technique for this is based on magnetic flux quantization: In 1989 Tate et. al [9], through the measurement of the London moment magnetic trapped flux, reported an anomalous Cooper pair mass excess in thin rotating Niobium superconductive rings:

$$
\Delta m=m^{*}-m=(94 \pm 21) \mathrm{eV}
$$

Here $m^{*}=1.000084(21) \times 2 m_{e}=1.023426(21) \mathrm{MeV}$ is the experimentally measured Cooper pair mass (with an accuracy of $21 \mathrm{ppm}$ ), and $m=0.999992 \times 2 m_{e}=$ $1.002331 \mathrm{MeV}$ is the theoretically expected Cooper pair mass including relativistic corrections. The above Cooper pair mass excess (or, equivalently, the slightly larger than expected measured magnetic field) has not been explained until now.

Motivated by the absence of any apparent solution of this disagreement in the existing literature, one of us (C. de Matos) formulated the conjecture that an additional 
gravitomagnetic term must be added to the Cooper pairs' canonical momentum:

$$
\vec{\pi}=m \vec{v}+e \vec{A}+m \vec{A}_{g}
$$

The strength of the extra term $m \vec{A}_{g}$ can then be estimated from the experimental data. The gravitomagnetic field strength $B_{g}=\left|\vec{B}_{g}\right|=\left|\nabla \times \vec{A}_{g}\right|$ required to account for the reported mass excess is 31 orders of magnitude larger than any gravitomagnetic field predicted by general relativity, based on the mass currents in the rotating ring [16, 17]. It can be expressed in terms of (8) as

$$
B_{g}=\frac{\Delta m}{m} 2 \omega=1.84 \times 10^{-4} \omega
$$

Here $\vec{\omega}$ is the superconductor's angular velocity. The physical interpretation of (10) is ambiguous. Either it can be understood as the gravitomagnetic London-type moment in rotating superconductors, or it can be associated with a deviation from the gravitomagnetic Larmor theorem [18], $B_{g}=2 \omega$, which would be revealed by anomalous Coriolis forces inside rotating superconductive cavities. These ambiguities will be further discussed and elucidated below. The most important question at this point is why the field $B_{g}$ is so much larger than expected, and why it is observed below a critical temperature only. In the following we will relate this to an effect produced by dark energy.

\section{Massive Photons in Superconductors}

The properties of superconductors (zero resistivity, Meissner effect, London moment, flux quantization, Josephson effect, etc.) can be understood from the spontaneous breaking of electromagnetic gauge invariance when the material is in the superconductive phase $[15,19]$. In quantum field theory, this symmetry breaking leads to massive photons via the Higgs mechanism. In this case the Maxwell equations transform to the so-called Maxwell-Proca equations, which are given by

$$
\begin{aligned}
\nabla \vec{E} & =\frac{\rho_{e}}{\epsilon_{0}}-\frac{1}{\lambda_{\gamma}^{2}} \phi, \\
\nabla \vec{B} & =0, \\
\nabla \times \vec{E} & =-\dot{\vec{B}}, \\
\nabla \times \vec{B} & =\mu_{0} \rho_{e} \vec{v}+\frac{1}{c^{2}} \dot{\vec{E}}-\frac{1}{\lambda_{\gamma}^{2}} \vec{A} .
\end{aligned}
$$

Here $\vec{E}$ is the electric field, $\vec{B}$ is the magnetic field, $\epsilon_{0}$ is the vacuum electric permittivity, $\mu_{0}=1 / \epsilon_{0} c^{2}$ is the vacuum magnetic permeability, $\phi$ is a scalar electric potential, $\vec{A}$ is a magnetic vector potential, $\rho_{e}$ is the Cooper pair condensate charge density, $\vec{v}$ is the Cooper pair velocity, and $\lambda_{\gamma}=\hbar / m_{\gamma} c$ is the photon's Compton wavelength, which is equal to the London penetration depth $\lambda_{L}=\sqrt{m / \mu_{o} e \rho_{e}}$.
Taking the curl of (14) and neglecting the term coming from the displacement current, we get the following equation for the magnetic field:

$$
\nabla^{2} \vec{B}-\frac{1}{\lambda_{\gamma}^{2}} \vec{B}=\frac{1}{\lambda_{L}^{2}} \frac{m}{e} 2 \vec{\omega} .
$$

Solving (15) for the 1-dimensional case, we obtain a magnetic field with a term that decays exponentially and another one that is proportional to the rotation frequency $\omega$. These are, respectively, the Meissner effect and the London moment

$$
B=C e^{-x / \lambda_{y}}+2 \omega \frac{m}{e}\left(\frac{\lambda_{\gamma}}{\lambda_{L}}\right)^{2} .
$$

Here $C$ is an arbitrary constant. Following Becker et al. [20] and London [21], the London moment is developed by a net current that is lagging behind the positively charged ion lattice. The Cooper pair current density shows in opposite direction than the angular velocity of the superconducting bulk. This is important as the London moment in all measurements, due to the negative charge of the Cooper pair, shows in the same direction as the angular velocity. Having

$$
\lambda_{\gamma}=\lambda_{L}
$$

we finally obtain the familiar expression

$$
B=C e^{-x / \lambda_{L}}-2 \omega \frac{m}{e} .
$$

The experiments of Tajmar et al. have been operating at temperatures of the order of $7 \mathrm{~K}$. At this temperature, the London penetration depth for Niobium is $\lambda_{L}=$ $\lambda_{L}(0) / \sqrt{1-\left(T / T_{c}\right)^{4}}=47.6 \mathrm{~nm}$ (assuming a critical temperature $T_{c}=9.25 \mathrm{~K}$, and a London penetration depth at $T=0$ of $\left.\lambda_{L}(0)=39 \mathrm{~nm}[22]\right)$. Substituting this value into (17), we deduce a typical value of the photon mass in Niobium

$$
m_{\gamma}=4.2 \mathrm{eV} \text {. }
$$

\section{Gravitational Maxwell-Proca Equations}

In analogy with the electromagnetic fields produced by a Cooper pair condensate, which are described by the set of Maxwell-Proca equations (11)-(14), we may write down analogous equations for gravity in the weak field approximation. These generate gravitoelectromagnetic fields according to a set of Einstein-Maxwell-Proca equations, with a massive graviton [23]

$$
\begin{aligned}
\nabla \vec{g} & =-\frac{\rho_{m}^{*}}{\epsilon_{0 g}}-\frac{1}{\lambda_{g}^{2}} \varphi_{g}, \\
\nabla \overrightarrow{B_{g}} & =0 \\
\nabla \times \vec{g} & =-\vec{B}_{g}, \\
\nabla \times \vec{B}_{g} & =-\mu_{0 g} \rho_{m}^{*} \vec{v}+\frac{1}{c^{2}} \dot{\vec{g}}-\frac{1}{\lambda_{g}^{2}} \vec{A}_{g} .
\end{aligned}
$$


Here $\vec{g}$ is the gravitational field, $\vec{B}_{g}$ is the gravitomagnetic field, $\epsilon_{0 g}=1 / 4 \pi G$ is the vacuum gravitational permittivity, $\mu_{0 g}=4 \pi G / c^{2}$ is the vacuum gravitomagnetic permeability, $\phi_{g}$ is the scalar gravitational potential, $\vec{A}_{g}$ is the gravitomagnetic vector potential, $\rho_{m}^{*}=\rho^{*} / c^{2}$ is the mass density of the gravitational condensate ( $\rho^{*}$ is the corresponding energy density), $\vec{v}$ is the velocity of the gravitational analogue of Cooper pairs, and $\lambda_{g}=\hbar / m_{g} c$ is the Compton wavelength of the graviton. At the moment, we will leave open the physical interpretation of the gravitational quantum condensate; we will come back to this in the next section. There are several theoretical approaches for the inclusion of a massive graviton in general relativity. Here we adopt the proposal from Deser and Waldron [24], to link the cosmological constant $\Lambda$ and the graviton mass, $m_{g}$, in a partially massless spin 2 theory in a de-Sitter background $(\Lambda>0)$, which describes 4 propagating degrees of freedom for the graviton corresponding to helicities $( \pm 2, \pm 1)$ (helicity 0 being unphysical). In this approach one has

$$
\frac{1}{\lambda_{g}^{2}}=\left(\frac{m_{g} c}{\hbar}\right)^{2}=\frac{2}{3} \Lambda
$$

At this particular (critical) value a novel local scalar gauge invariance appears that is responsible for the elimination of the helicity 0 excitation. In this theory in the limit of a massless field, $m_{g}=0$, the classical two degrees of freedom \pm 2 are recovered, and in the interval $0<m_{g}^{2}<2 \hbar^{2} \Lambda / 3 c^{2}$ the theory is unstable. Note that the above graviton mass is tiny. $\lambda_{g} / c$ is of the same order of magnitude as the current age of the universe, hence we should not expect any significant modification with respect to the presently experimentally verified laws of gravity from such a tiny postulated graviton mass.

In the 1-dimensional case we obtain the solution of (23) as

$$
B_{g}=C_{g} e^{-x / \lambda_{g}}+2 \omega\left(\frac{\lambda_{g}}{\lambda_{L}}\right)^{2},
$$

where $C_{g}$ is an arbitrary constant and

$$
\lambda_{L}=\frac{1}{\sqrt{\mu_{0_{g}} \rho_{m}^{*}}}
$$

is the gravitational analogue of the London penetration depth. In (25) the $x$-dependence of the first term (the gravitational Meissner effect) can be neglected due to the huge $\lambda_{g}$ assumed. The term of interest is the second term, the gravitomagnetic London-type moment, which can potentially lead to measurable effects, depending on what is assumed for $\rho^{*}$. The basic idea in the following is to associate $\rho^{*}$ with dark energy.

\section{Dark Energy in a Superconductor}

So far the Einstein-Maxwell-Proca equations considered in the previous section were not coupled to the electrodynamics of Cooper pairs. We now consider possible interactions, based on the model of dark energy as proposed by Beck and Mackey in [6].

A nonvanishing cosmological constant (CC) $\Lambda$ can be interpreted in terms of a non-vanishing vacuum energy density

$$
\rho_{v a c}=\frac{c^{4}}{8 \pi G} \Lambda
$$

which corresponds to dark energy with equation of state $w=-1$. The small astronomically observed value of the CC, $\Lambda=1.29 \times 10^{-52} \mathrm{~m}^{-2}[1]$, and its origin remain a deep mystery. This is often called the CC problem, since with a cutoff at the Planck scale the vacuum energy density expected from quantum field theories should be larger by a factor of the order $10^{120}$, in complete contradiction with the observed value. To solve the CC problem, in [6] a model of dark energy was suggested that is based on bosonic vacuum fluctuations creating a small amount of vacuum energy density. One assumes that photons (or any other bosons), with zeropoint energy $\epsilon=(1 / 2) h v$, can exist in two different phases: a gravitationally active phase where the zeropoint fluctuations contribute to the cosmological constant $\Lambda$, and a gravitationally inactive phase where they do not contribute to $\Lambda[6,25-27]$. This is described in [6] by a Ginzburg-Landau type of theory. As shown in [6], this type of model of dark energy can lead to measurable effects in superconductors, via a possible interaction with the Cooper pairs in the superconductor.

Whereas details of this dark energy model are described in [6], let us here summarize the main points of that model which are important for our experimental predictions in the following sections. In superconductors, the number density of superconducting electrons is described by the square of a macroscopic wave function $\left|\Psi_{s}\right|^{2}$. Similarly, in the dark energy model [6] the number density of gravitationally active photons (with vacuum fluctuations contributing to $\Lambda$ ) is described by the square of yet another wave function $\left|\Psi_{g}\right|^{2}$. Both theories are described by Ginzburg-Landau potentials of the form

$$
\begin{aligned}
& F_{s}=a\left|\Psi_{s}\right|^{2}+b\left|\Psi_{s}\right|^{4}, \\
& F_{g}=a^{\prime}\left|\Psi_{g}\right|^{2}+b^{\prime}\left|\Psi_{g}\right|^{4},
\end{aligned}
$$

and both models exhibit a phase transition, the former one at the critical temperature $T_{c}$ of the superconductor and the latter one at a critical frequency $v_{c}$ above which vacuum fluctuations are gravitationally inactive. The finite dark energy density as generated by gravitationally active vacuum fluctuations in the model [6] leads to an accelerated expansion of the universe on large scales as a consequence of the Friedmann equations. However, the model also allows for observable effects of dark energy on much smaller, laboratory scales, in the interior of superconductors, due to the fact that the two Ginzburg-Landau potentials can 
influence each other. In [6] phases $\Phi_{s}$ and $\Phi_{g}$ of the macroscopic wave functions are introduced by writing

$$
\begin{aligned}
& \Psi_{s}=\left|\Psi_{s}\right| e^{i \Phi_{s},} \\
& \Psi_{g}=\left|\Psi_{g}\right| e^{i \Phi_{g} .}
\end{aligned}
$$

Gradients in the phases of the wave functions induce generalized currents, which in principle can be experimentally measured. For more details, see [6].

Here we introduce the following additional hypotheses with respect to the original model in [6], which, as we will see later, are consistent with the experimental observations in rotating superconductors.

(1) Like normal photons (with energy $\epsilon=h \nu$ ), the gravitationally active photons (with zeropoint energy $\epsilon=$ $(1 / 2) h v)$ acquire mass in a superconductor due to the spontaneous breaking of gauge invariance. In the following we call these spin 1 bosons graviphotons. In Niobium their mass is approximately $\sim 4 \mathrm{eV}$, as we saw previously in (19).

(2) The transition between the two graviphoton's phases (gravitationally active, versus gravitationally inactive) occurs at the critical temperature $T_{c}$ of the superconductor, which defines a cutoff frequency $v_{c}$ of zeropoint fluctuations specific to each superconductive material: $h v_{c} \sim k T_{c}$.

(3) Graviphotons can form weakly bounded states with Cooper pairs, increasing their mass slightly from $m$ to $\tilde{m}$. The binding energy is $\epsilon_{c}=\mu c^{2}$ and

$$
\tilde{m}=m+m_{\gamma}-\mu .
$$

(4) Since the graviphotons are bounded to the Cooper pairs, their zeropoint energies form a condensate capable of the gravitoelectrodynamic properties of superconductive cavities.

Beck and Mackey's Ginzburg-Landau-like theory leads to a finite dark energy density dependent on the frequency cutoff $v_{c}$ of vacuum fluctuations:

$$
\rho^{*}=\frac{1}{2} \frac{\pi h}{c^{3}} v_{c}^{4} .
$$

In vacuum one may put $\rho^{*}=\rho_{v a c}$, from which the cosmological cutoff frequency $v_{c c}$ is estimated as

$$
v_{c c} \simeq 2.01 \mathrm{THz} .
$$

The corresponding "cosmological" quantum of energy is.

$$
\epsilon_{c c}=h \nu_{c c}=8.32 \mathrm{meV} .
$$

Note that this is the same order of magnitude as in dark energy models introduced 20 years ago [28]; the main difference being that our dark energy field now has the new property of being able to interact with superconducting electrons in superconducting matter.

In the interior of superconductors, according to Assumption 2, the effective cutoff frequency can be different from that in (32). This is due to interaction effects between the two Ginzburg-Landau potentials (that of the superconducting electrons and that of the dark energy model) [6]. The effect can be seen in analogy to polarization effects of ordinary electromagnetic fields in matter. In matter the electric field energy density is different as compared to the vacuum. Similarly, in superconductors the effectice dark energy density (represented by gravitationally active zeropoint fluctuations) can be different as compared to the vacuum. Our model allows for the gravitational analogue of polarization.

An experimental effort is currently taking place at University College London and the University of Cambridge to measure the cosmological cutoff frequency through the measurement of the spectral density of the noise current in resistively shunted Josephson junctions, extending earlier measurements of Koch et al. [29].

In [6] the formal attribution of a temperature $T$ to the graviphotons is done by comparing their zeropoint energy with the energy of ordinary photons in a bath at temperature $T$ :

$$
\frac{1}{2} h \nu=\frac{h v}{e^{h v / k T}-1} .
$$

This condition is equivalent to

$$
h v=\ln 3 k T .
$$

Substituting the critical transition temperature $T_{c}$ specific to a given superconductive material into (35), we can calculate the critical frequency characteristic for this material:

$$
v_{c}=\ln 3 \frac{k T_{c}}{h} .
$$

For example, for Niobium with $T_{c}=9.25 \mathrm{~K}$ we get $v_{c}=0.212$ $\mathrm{THz}$. If we use the cosmological cutoff frequency in (36) we find the cosmological critical temperature $T_{c c}$ :

$$
T_{c c}=87.49 \mathrm{~K} .
$$

This temperature is a characteristic of the BSCCO High- $T_{c}$ superconductor.

\section{Graviphotonic Effect in Accelerated Superconductors}

Let us now come to our physical explanation of the observed experimental effects, using the dark energy model of the previous section. In Tajmar's type- 1 experiments, a strong angular acceleration applied to the superconductive ring can break the bound between a Cooper pair and its associated graviphoton. In that process the Cooper pair looses the mass $m_{\gamma} \sim 4 \mathrm{eV}$, which by reaction effectively produces an acceleration, on the Cooper pairs, in the opposite direction of the applied acceleration. This generates a macroscopic measurable acceleration, since a coherent patch of a large number of graviphotons is simultaneously expelled out of the superconductor and absorbed by the vacuum. Our hypothesis is that this produces the measured induced acceleration in Tajmar's experiment, as can be deduced by applying Newton's law of action reaction to the system formed by a Cooper pair and the graviphoton:

$$
-f_{\text {Graviphoton }}=-m_{\gamma} \dot{\omega} R=m g=f_{\text {CooperPair }} .
$$


Here $R \sim 0.07[\mathrm{~m}]$ is the superconductive Niobium ring radius (in Tajmar's experiment), $f_{\text {Graviphoton }}$ is the force applied on the massive graviphotons due to the angular acceleration communicated to the superconductive ring, and $f_{\text {CooperPair }}$ is the corresponding reaction force experienced by the Cooper pair, $\dot{\omega}$ is the superconductor's angular acceleration measured in $s^{-2}$, and $g$ is the produced acceleration at distance $R$. From (38), we find the coupling

$$
\frac{g}{\dot{\omega}}=-\frac{m_{\gamma}}{m} R
$$

Substituting into (39) the value of the graviphoton mass for Niobium found in (19), we obtain at distance $R=$ $0.07 \mathrm{~m}$ the value $g / \dot{\omega} \approx-2.9 \times 10^{-7} \mathrm{~m}$. But the actual acceleration measurements are done with accelerometers that are positioned at the smaller distance $r=0.036 \mathrm{~m}$, where according to (22) we expect to see an induced field that is smaller by a factor $r / R=0.51$. We thus obtain at $r=0.036 \mathrm{~m}$ the theoretical prediction

$$
\frac{g}{\dot{\omega}} \approx-1.49 \times 10^{-7} \mathrm{~m} .
$$

This is in excellent agreement with the experimentally measured value as given in $(2), g / \dot{\omega}=-(1.51 \pm 0.04) \times$ $10^{-7} \mathrm{~m}$.

If the superconductive ring rotates with constant angular velocity and the temperature $T$ is decreased below $T_{c}$, then spontaneously Cooper pairs form and these immediately absorb graviphotons out of the vacuum. By this process their mass increases, and an acceleration signal is detected. Similarly, if $T$ is increased above $T_{c}$, then the material immediately looses the graviphotons to the vacuum, the Cooper pair mass decreases, and an opposite acceleration signal is measured, as reported in Tajmar's experiment.

We suggest to call this coherent emission of graviphotons by accelerated superconductors the "graviphotonic effect."

\section{Gravitomagnetic London Moment in Rotating Superconductors}

Let us first provide a short derivation of the ordinary electromagnetic London moment-after that we will proceed to the gravitomagnetic London moment. The Cooper pairs in a superconductor can be regarded as a condensate described by the wave function $\Psi_{s}$ :

$$
\Psi_{s}=\rho_{e}^{1 / 2} e^{i \phi_{s}} .
$$

Here $\rho_{e}$ is equal to the electric charge density of the Cooper pair condensate, and $\phi_{s}$ is the phase of the wave function. The canonical momentum of the Cooper pairs, $\vec{\pi}$, is proportional to the gradient of the phase of the wave function

$$
\vec{\pi} \sim \nabla \phi_{s}
$$

Explicitly, the canonical momentum contains a mechanical and a magnetic term:

$$
\vec{\pi}=m \vec{v}+e \vec{A}
$$

Here again $m \approx 2 m_{e}$ is the Cooper pair mass and $e$ the Cooper pair charge (i.e., twice the electron charge), $\vec{v}$ is the Cooper pair's velocity with respect to an inertial reference frame attached to the laboratory, and $\vec{A}$ is the magnetic vector potential. From (42) we deduce that the curl of the Cooper pairs' canonical momentum is always zero:

$$
\nabla \times \vec{\pi}=0
$$

Taking the curl of (43) and using the fact that $\vec{v}=\vec{r} \times \vec{\omega}$ and $\vec{B}=\nabla \times \vec{A}$ we obtain the London moment

$$
\vec{B}=-2 \frac{m}{e} \vec{\omega}
$$

In close analogy to the above derivation, let us now proceed to a gravitomagnetic London moment as produced by dark energy. As seen before, our central hypothesis is that the gravitational quantum condensate is related to dark energy. We may assume that cosmological quanta of energy $h v_{c c}$ manifest themselves as massive particles of mass $\mu$ in the superconductor:

$$
\mu c^{2}=h v_{c c}
$$

These can be regarded as the gravitational analogues of the Cooper pairs in our model (note the similarity with typical neutrino mass scales). We expect them to be strongly correlated to ordinary Cooper pairs and to rotate with the superconductor. In close analogy to (43) (i.e., replacing $m \rightarrow$ $\mu, e \rightarrow-m, \vec{A} \rightarrow \vec{A}_{g}$ ) we now consider the gravitational canonical momentum

$$
\vec{\pi}_{g}=\mu \vec{v}-m \vec{A}_{g}-m_{\gamma} \vec{A}_{g}
$$

where the term $-m \vec{A}_{g}$ describes the interaction with the ordinary Cooper pairs of mass $m$. Assuming that the dark energy quanta, the ordinary Cooper pairs and the graviphotons are described by the same macroscopic wave function (i.e., implementing the assumption of phase synchronisation as in [6]) we again obtain $\vec{\pi}_{g} \sim \nabla \phi_{g}$ where $\phi_{g}$ is the gravitational phase, hence

$$
\nabla \times \vec{\pi}_{g}=0
$$

By taking the curl in (47) we thus obtain the gravitomagnetic London moment

$$
\vec{B}_{g}=2 \frac{\mu}{m+m_{\gamma}} \vec{\omega}
$$

Putting in $m+m_{\gamma} \approx m \approx 2 m_{e}$ and $\mu c^{2}=h v_{c c}$ we obtain the numerical value

$$
B_{g}=1.62 \times 10^{-8} \omega
$$

This theoretical prediction is consistent with Tajmar's type2 measurements, $(6)$, where $B_{g} / \omega$ was measured to be of the order $10^{-8}[10,12]$. 


\section{Nonclassical Inertia in Superconductive Cavities}

From the Einstein-Maxwell-Proca equations of our electromagnetic model of dark energy with massive bosons we can derive the inertial properties of a superconductive cavity. Taking the gradient of (20), and the curl of (23), and solving the resulting differential equations for the 1dimensional case we find, respectively, a form of the principle of equivalence and of the gravitomagnetic Larmor Theorem [18] in superconductive cavities:

$$
\begin{aligned}
\vec{g} & =-\vec{a} \mu_{0 g} \rho_{m}^{*} \lambda_{g}^{2}, \\
\vec{B}_{g} & =2 \vec{\omega} \mu_{0 g} \rho_{m}^{*} \lambda_{g}^{2} .
\end{aligned}
$$

Here $\vec{a}$ is an acceleration communicated to the superconductive cavity, and $\vec{g}$ is an acceleration measured inside the superconductive cavity. For the derivation of (51) we assumed the case of a homogeneous field $\vec{g}$ and used the formulas $\vec{g}=-\nabla \phi_{g}$ and $\rho_{m}^{*} \vec{a}=-\nabla\left(\rho_{m}^{*} c^{2}\right)$. For (52) we used Becker's argument that the Cooper pairs are lagging behind the lattice so that the current is flowing in the opposite direction of $\omega$. We now express the coupling $\chi=B_{g} / \omega$ between the gravitomagnetic field and the superconductor's angular velocity directly as a function of the dark energy density $\rho^{*}$ contained in the superconductor through the substitution of $\rho_{m}^{*}=\rho^{*} / c^{2}$ in (52):

$$
\chi=\frac{8 \pi G}{c^{4}} \rho^{*} \lambda_{g}^{2}
$$

Substituting (27) and (24) into (53) and rearranging we obtain

$$
\chi=\frac{3}{2} \frac{\rho^{*}}{\rho_{v a c}} .
$$

Substituting (27), (31), and (36) into (54) we obtain

$$
\chi=\frac{3 \ln ^{4} 3}{4 \pi} \frac{k^{4} G}{c^{7} \hbar^{3} \Lambda} T_{c}^{4} .
$$

Remarkably, this equation connects the five fundamental constants of nature $k, G, c, \hbar, \Lambda$ with measurable quantities in a superconductor, $\chi$ and $T_{c}$.

We may define a Planck-Einstein temperature $T_{\mathrm{PE}}$ as

$$
T_{\mathrm{PE}}=\frac{1}{k}\left(\frac{c^{7} \hbar^{3} \Lambda}{G}\right)^{1 / 4}=60.71 \mathrm{~K}
$$

Equation(55) can then be written as [30]

$$
\chi=\frac{3 \ln ^{4} 3}{4 \pi}\left(\frac{T_{c}}{T_{\mathrm{PE}}}\right)^{4} .
$$

Substituting the critical transition temperature of Niobium, $T_{c}=9.25 \mathrm{~K}$, into (57) we find the following coupling between the gravitomagnetic field and the angular velocity of a rotating superconductive Niobium ring:

$$
\chi=1.87 \times 10^{-4}
$$

TABle 1: Predicted coupling $\chi$ between the gravitomagnetic field and the angular velocity for different superconductive materials.

\begin{tabular}{lcc}
\hline Superconductive material & $T_{c}[\mathrm{~K}]$ & $\chi$ \\
\hline $\mathrm{Al}$ & 1.18 & $4.96 \times 10^{-8}$ \\
$\mathrm{In}$ & 3.41 & $3.46 \times 10^{-6}$ \\
$\mathrm{Sn}$ & 3.72 & $4.90 \times 10^{-6}$ \\
$\mathrm{~Pb}$ & 7.2 & $6.88 \times 10^{-5}$ \\
$\mathrm{Nb}$ & 9.25 & $1.87 \times 10^{-4}$ \\
$\mathrm{High}-T_{c}$ & 79.06 & 1 \\
$\mathrm{BSCCO}$ & 87.5 & 1.5 \\
YBCO & 94.0 & 2 \\
\hline
\end{tabular}

This coupling describes the effect of gravitationally active zeropoint energy within the superconductor. The previous theoretically predicted value is extremely close to the measured value in Cabrera and Tate's experiment, (10),

$$
\chi=2 \frac{\Delta m}{m}=1.84 \times 10^{-4} \text {. }
$$

Let us evaluate the theoretically predicted coupling for various types of superconductors, starting with Aluminium and ending with High- $T_{c}$ superconductors like YBCO.

We note that for $\mathrm{YBCO}$, with $T_{c}=94.0 \mathrm{~K}$, we recover the classical gravitational Larmor theorem [18]:

$$
B_{g}=2 \omega
$$

From Table 1 we conclude that the effective laws of inertia in superconductive cavities deviate from the laws of classical mechanics, recovering however the classical regime in the limit of YBCO cavities.

At this point one remark is at order. Our theoretical derivation presented in this paper strictly speaking holds only for conventional low- $T_{c}$ superconductors, because we are using simple Ginzburg-Landau models and BCS-type of arguments for both the superconductor and the dark energy model [6]. A high- $T_{c}$ superconductor, however, is not decribed by such a simple theory. Whether a high- $T_{c}$ superconductor can form bounded states with graviphotons, thus leading to our predicted effects, is theoretically unclear. The measurements of Tajmar in [10] did not show any signal above the noise level for high- $T_{c}$ superconductors. Moreover, the London moment measured for high- $T_{c}$ superconductors seems not to show any anomalous behavior within the experimental precision [31]. It is likely that our theory holds for conventional superconductors only, where the coupling $\chi$ is small.

Our approach raises interesting perspectives for future experiments.

(1) Measurements of the Coriolis force in rotating low- $T_{c}$ superconductive cavities should show a deviation from the predictions of classical mechanics.

(2) If in Tajmar's type-2 experiment the laser-gyro is mechanically attached to the rotating Niobium superconductive ring we should find the "gravito-angular" coupling, 
$\chi=1.84 \times 10^{-4}$, associated with the Cabrera and Tate Cooper pair mass excess. If instead the laser-gyro is mechanically decoupled from the rotating superconductive ring, which correspond to the current configuration, we should observe the gravitomagnetic London moment characterised by a coupling $\chi^{\prime}=1.61 \times 10^{-8},(6)$, reported in Tajmar's recent experiments.

(3) The dependence of the coupling $\chi$ on various superconducting materials should be further investigated. High$T_{c}$ superconductors may require a different gravitomagnetic theory.

\section{Discussion}

Let us end this paper with some general remarks. General Relativity is founded on the Principle of Equivalence, which rests on the equality between the inertial and the gravitational mass of any physical system. The Principle of General Covariance (PGC) is an alternative version of the principle of equivalence [32], which is very appropriate to investigate the field equations for electromagnetism and gravitation. It states that a physical equation holds in a general gravitational field if the two following conditions are met:

(1) the equation holds in the absence of gravitation; that is, it agrees with the laws of special relativity when the metric tensor $g_{\alpha \beta}$ equals the Minkowsky tensor $\eta_{\alpha \beta}$ and when the affine connection $\Gamma_{\beta \gamma}^{\alpha}$ vanishes.

(2) the equation is generally covariant; that is, it preserves its form under a general coordinate transformation $x \rightarrow x^{\prime}$.

Any physical principle such as the PGC, which takes the form of an invariance principle but whose content is actually limited to a restriction on the interaction of one particular field, is called a dynamic symmetry. Local gauge invariance, which governs the electromagnetic interaction, is an important example of a dynamic symmetry. We can actually say that the Principle of General Covariance in general relativity is the analogue of the Principle of Gauge Invariance in electrodynamics. The breaking of gauge invariance leads to superconducting states. The breaking of general covariance leads to nonconservation of energy momentum (in the covariant sense) [32]. In this context it is interesting to see that our gravitational dark energy quantum condensate is related to zeropoint fluctuations, for which energy is certainly not conserved.

There are several possibilities to make the above ideas on the breaking of PGC more concrete. One is based on geometric algebra and gauge theories of gravity [33]. In this approach the spontaneous breaking of gauge invariance of one of the gauge groups underlying gravity would stand in relation to the spontaneous breaking of covariance [34]. Quantum violations of the equivalence principle have also been discussed (in a black hole context) in [35]. The statistics of dark energy quantum fluctuations in superconductors is further discussed in [36].

\section{Conclusion}

In this paper we have investigated in detail the possibility that the dark energy of the universe may interact with Cooper pairs in superconductors, thus leading to effects that can be observed in the laboratory. Whether or not such an interaction is a realistic assumption depends very much on the dark energy model considered. The electromagnetic dark energy model of Beck and Mackey [6], and its further development as presented in the current paper, naturally contains such an interaction.

There are first experimental hints that one might be on the right track with these types of theoretical models. The graviphotonic effect, the gravitomagnetic London moment, and nonclassical inertia in rotating superconductive cavities are three different experimentally observed effects which can all be explained by the proposed model of dark energynot only qualitatively but also quantitatively. The model ultimately relies on the spontaneous breaking of gauge invariance and the spontaneous breaking of the principle of general covariance in the interior of superconductors.

The considerations presented in this paper, if confirmed by further independent experiments, would imply that the dark energy of the universe produces measurable effects not only on cosmological scales but also in the interior and the vicinity of superconductors. This opens up the way for a variety of new possible laboratory experiments testing the nature of dark energy and constraining the interaction strength with Cooper pairs. In our model gravitationally active vacuum fluctuations underlying dark energy lead to a strong enhancement of gravitomagnetic fields, in quantitative agreement with the anomalies seen in the experiments of Tate et al. [9] and Tajmar et al. [10, 11].

\section{Acknowledgment}

The authors would like to thank Dr. Martin Tajmar for useful comments on an early version of this paper.

\section{References}

[1] D. N. Spergel, L. Verde, H. V. Peiris, et al., "First-year Wilkinson Microwave Anisotropy Probe (WMAP) observations: determination of cosmological parameters," Astrophysical Journal, Supplement Series, vol. 148, no. 1, pp. 175-194, 2003.

[2] P. J. E. Peebles and B. Ratra, "The cosmological constant and dark energy," Reviews of Modern Physics, vol. 75, no. 2, pp. 559-606, 2003.

[3] E. J. Copeland, M. Sami, and S. Tsujikawa, "Dynamics of dark energy," International Journal of Modern Physics D, vol. 15, no. 11, pp. 1753-1935, 2006.

[4] T. Padmanabhan, "Cosmological Constant-the Weight of the Vacuum," Physics Reports, vol. 380, pp. 235-320, 2003.

[5] D. J. Kapner, T. S. Cook, E. G. Adelberger, et al., "Tests of the gravitational inverse-square law below the dark-energy length scale," Physical Review Letters, vol. 98, no. 2, Article ID 021101, 2007. 
[6] C. Beck and M. C. Mackey, "Electromagnetic dark energy," International Journal of Modern Physics D, vol. 17, no. 1, pp. 71-80, 2008.

[7] S. Alexander, M. Mbonye, and J. Moffat, "The gravitational instability of the vacuum: insight into the cosmological constant problem," http://arxiv.org/abs/hep-th/0406202.

[8] V. Jejjala, M. Kavic, and D. Minic, "Fine structure of dark energy and new physics," Advances in High Energy Physics, vol. 2007, Article ID 21586, 14 pages, 2007.

[9] J. Tate, B. Cabrera, S. B. Felch, and J. T. Anderson, "Precise determination of the Cooper-pair mass," Physical Review Letters, vol. 62, no. 8, pp. 845-848, 1989.

[10] M. Tajmar, F. Plesescu, B. Seifert, and K. Marhold, "Measurement of gravitomagnetic and acceleration fields around a rotating superconductor," in Proceedings of the Conference on Space Technology and Applications International Forum (STAIF '07), vol. 880 of AIP Conference Proceedings, pp. 10711082, 2007.

[11] M. Tajmar, F. Plesescu, K. Marhold, and C. J. de Matos, "Experimental detection of the gravitomagnetic London moment," http://arxiv.org/abs/gr-qc/0603033.

[12] M. Tajmar, F. Plesescu, B. Seifert, R. Schnitzer, and I. Vasiljevich, "Search for frame dragging in the vicinity of spinning superconductors," in Proceedings of the 2nd International Conference on Time and Matter, vol. 49, Nova Gorica, Slovenia, 2008.

[13] C. Kiefer and C. Weber, "On the interaction of mesoscopic quantum systems with gravity," Annalen der Physik, vol. 14, no. 4, pp. 253-278, 2005.

[14] C. J. de Matos and M. Tajmar, "Gravitomagnetic London moment and the graviton mass inside a superconductor," Physica C, vol. 432, no. 3-4, pp. 167-172, 2005.

[15] S. Weinberg, The Quantum Theory of Fields, vol. 2, Cambridge University Press, Cambridge, UK, 1996.

[16] M. Tajmar and C. J. de Matos, "Gravitomagnetic field of a rotating superconductor and of a rotating superfluid," Physica C, vol. 385, no. 4, pp. 551-554, 2003.

[17] M. Tajmar and C. J. de Matos, "Extended analysis of gravitomagnetic fields in rotating superconductors and superfluids," Physica C, vol. 420, no. 1-2, pp. 56-60, 2005.

[18] B. Mashhoon, "On the gravitational analogue of Larmor's theorem," Physics Letters A, vol. 173, no. 4-5, pp. 347-354, 1993.

[19] L. H. Ryder, Quantum Field Theory, Cambridge University Press, Cambridge, UK, 2nd edition, 1996.

[20] R. Becker, G. Heller, and F. Sauter, "Über die Stromverteilung in einer supraleitenden Kugel," Zeitschrift für Physik, vol. 85, no. 11-12, pp. 772-787, 1933.

[21] F. London, Superfluids, John Wiley \& Sons, New York, NY, USA, 1950.

[22] C. Kittel, Introduction to Solid State Physics, John Wiley \& Sons, New York, NY, USA, 8th edition, 2005.

[23] J. Argyris and C. Ciubotariu, "Massive gravitons in general relativity," Australian Journal of Physics, vol. 50, no. 5, pp. 879891, 1997.

[24] S. Deser and A. Waldron, "Stability of massive cosmological gravitons," Physics Letters B, vol. 508, no. 3-4, pp. 347-353, 2001.

[25] C. Beck and M. C. Mackey, "Could dark energy be measured in the lab?" Physics Letters B, vol. 605, no. 3-4, pp. 295-300, 2005.

[26] C. Beck, "Laboratory tests on dark energy," Journal of Physics: Conference Series, vol. 31, no. 1, pp. 123-130, 2006.
[27] C. Beck and M. C. Mackey, "Measurability of vacuum fluctuations and dark energy," Physica A, vol. 379, no. 1, pp. 101-110, 2007.

[28] B. Rhatra and P. J. E. Peebles, "Cosmology with a time-variable cosmological constant," The Astrophysical Journal Letters, vol. 325, p. L17, 1988.

[29] R. H. Koch, D. J. van Harlingen, and J. Clarke, "Quantumnoise theory for the resistively shunted Josephson junction," Physical Review Letters, vol. 45, no. 26, pp. 2132-2135, 1980.

[30] C. J. de Matos, "Electromagnetic dark energy and gravitoelectrodynamics of superconductors," Physica C, vol. 468, no. 3, pp. 210-213, 2008.

[31] A. A. Verheijen, J. M. van Ruitenbeek, R. de Bruyn Ouboter, and L. J. de Jongh, "Measurement of the London moment in two high-temperature superconductors," Nature, vol. 345, no. 6274, pp. 418-419, 1990.

[32] S. Weinberg, Gravitation and Cosmology: Principles and Applications of the General Theory of Relativity, John Wiley \& Sons, New York, NY, USA, 1972.

[33] A. Lasenby, C. Doran, and S. Gull, "Gravity, gauge theories and geometric algebra," Philosophical Transactions of the Royal Society A, vol. 356, no. 1737, pp. 487-582, 1998.

[34] A. Lasenby, private communication.

[35] C. Doran, A. Lasenby, S. Dolan, and I. Hinder, "Fermion absorption cross section of a Schwarzschild black hole," Physical Review D, vol. 71, no. 12, Article ID 124020, 6 pages, 2005.

[36] C. Beck and C. J. de Matos, "The dark energy scale in superconductors: innovative theoretical and experimental concepts," http://arxiv.org/abs/0709.2373. 

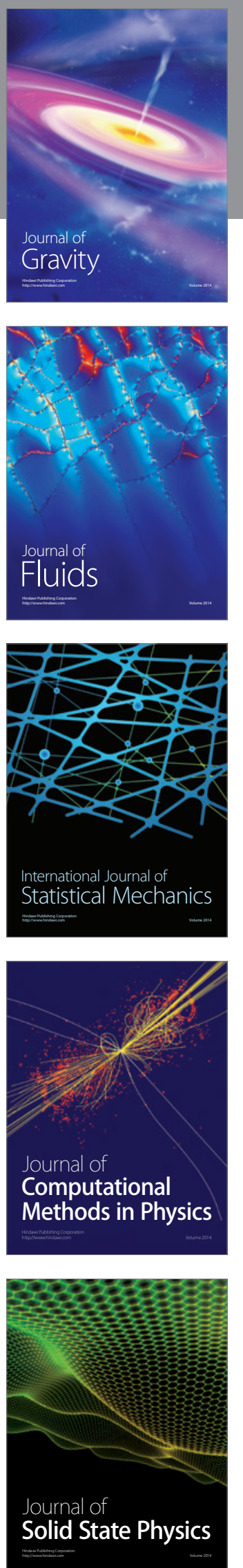

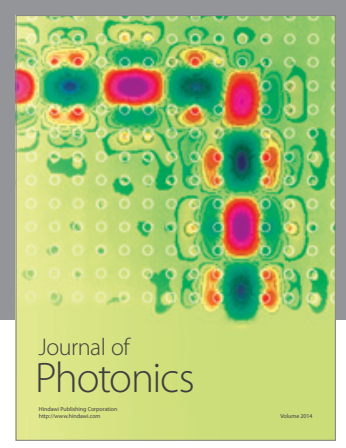

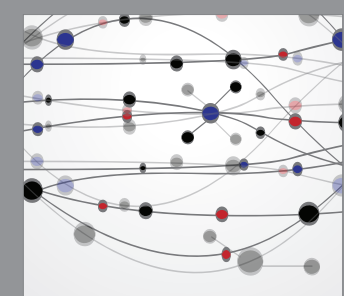

The Scientific World Journal
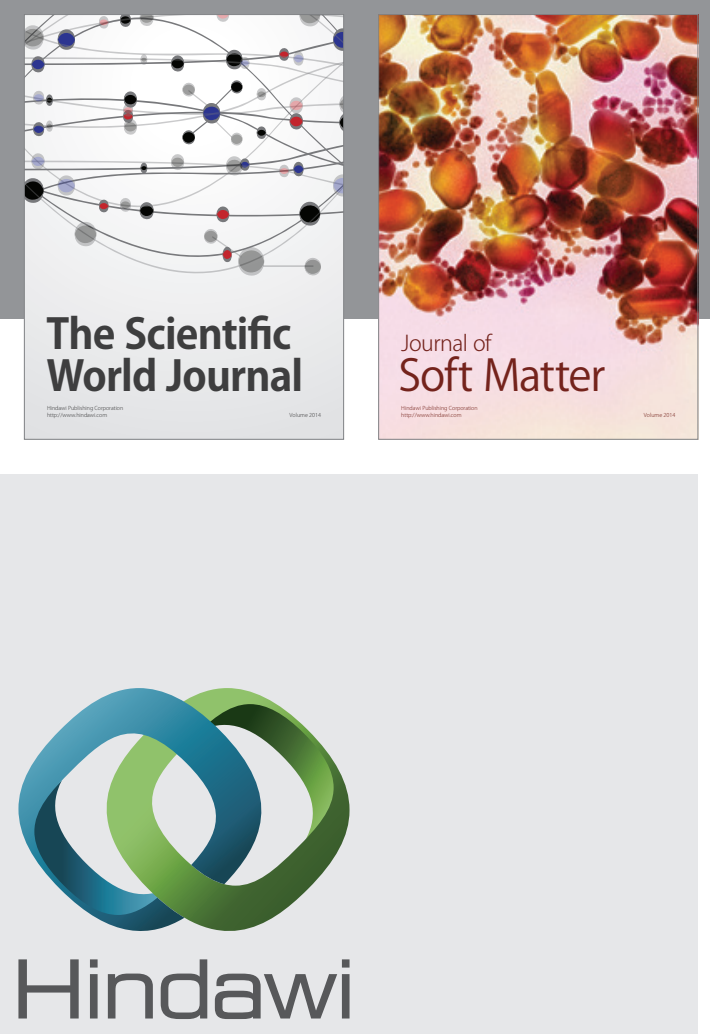

Submit your manuscripts at

http://www.hindawi.com
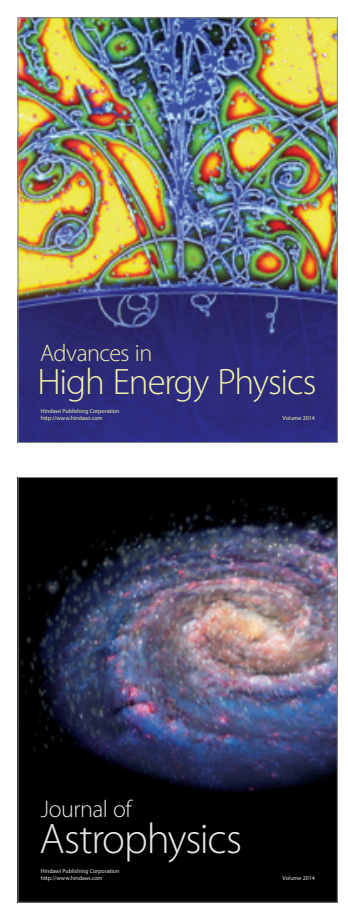
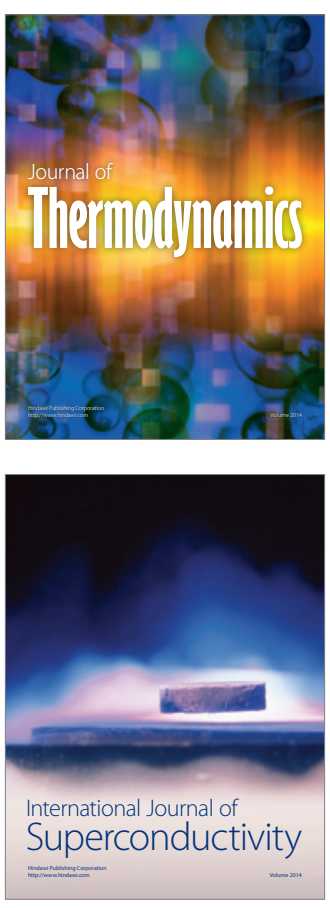
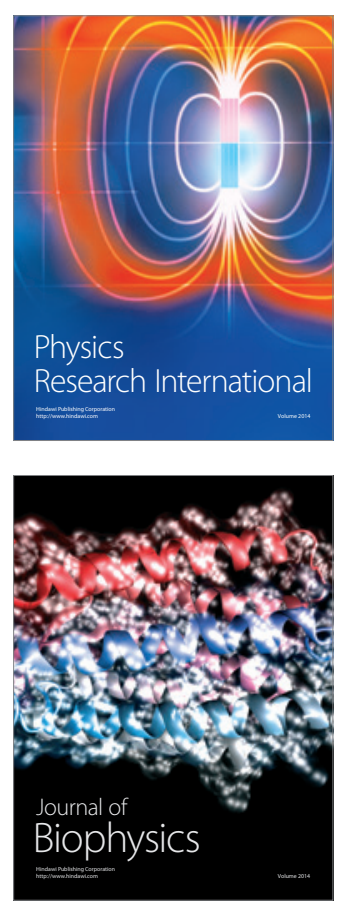
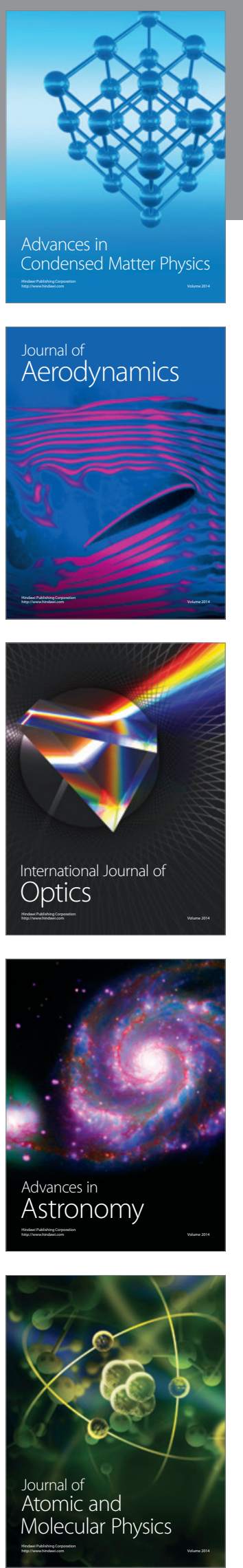